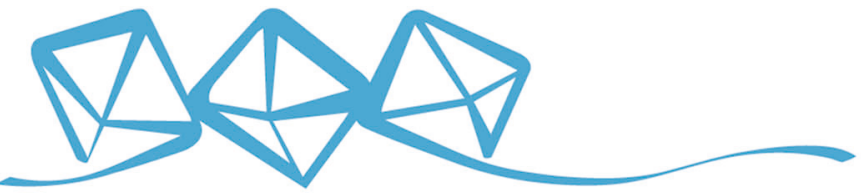 COMMUNICATIONS MATERIALS
}

ARTICLE

Check for updates

\section{Additive manufacturing assisted van der Waals integration of 3D/3D hierarchically functional nanostructures}

Jui-Han Fu'1,2,3,7, Ang-Yu Lu'4,7, Nathan J. Madden ${ }^{5,6}$, Christine C. Wu ${ }^{3}$, Yen-Chang Chen ${ }^{3}$, Ming-Hui Chiu ${ }^{1,2,4}$, Khalid Hattar ${ }^{5}$, Jessica A. Krogstad (10 6 , Stanley S. Chou ${ }^{5}$, Lain-Jong Li (i) ${ }^{1}$, Jing Kong ${ }^{4}$ \& Vincent Tung (10 1,2网

Van der Waals (vdW) integration, in which pre-engineered two-dimensional building blocks are physically assembled together in a chosen sequence through weak vdW interactions, holds promise toward previously unattainable applications. However, when extended to create 3D/3D monoliths, the lack of physical bonding coupled with the inherent rigidity and surface roughness between 3D building blocks makes it challenging for broader implementation of composites, catalysis, and energy applications. Here we demonstrate that electrostatically exfoliated two-dimensional layered materials can be additively manufactured to create complex layouts with selectively engineered composition in both lateral and vertical directions. Subsequent room-temperature dewetting creates non-covalent hinges through folded edges to concurrently interlock and nanostructure the two-dimensional inks into 3D building blocks. The result is the 3D/3D vdW mono- and heterostructures that are mechanically robust, electrically conductive, electrochemically active over a broad $\mathrm{pH}$ range and even radiation tolerant in nature.

\footnotetext{
${ }^{1}$ Physical Sciences and Engineering Division, King Abdullah University of Science and Technology (KAUST), KAUST Solar Centre, Thuwal 23955-6900, Saudi Arabia. ${ }^{2}$ Physical Science and Engineering Division, King Abdullah University of Science and Technology, Thuwal 23955-6900, Saudi Arabia. ${ }^{3}$ Molecular Foundry, Lawrence Berkeley National Lab, Berkeley, CA 94720, USA. ${ }^{4}$ Department of Electrical Engineering, Massachusetts Institute of Technology, Cambridge, MA 02139, USA. ${ }^{5}$ Sandia National Laboratories, Albuquerque, NM 87185, USA. ${ }^{6}$ Department of Materials Science and Engineering, University of Illinois, Urbana-Champaign, Urbana, IL 61801, USA. ${ }^{7}$ These authors contributed equally: Jui-Han Fu, Ang-Yu Lu. ${ }^{凶}$ email: vincent.tung@kaust.edu.sa
} 
$\mathrm{V}$ an der Waals (vdW) integration allows highly disparate material building blocks with pre-engineered functionalities to be physically assembled together through weak $\mathrm{vdW}$ interactions. This represents a potentially high throughput, and low-energy material-integration approach ${ }^{1-6}$. Notable innovations include bulk heterojunction in polymer photovoltaics ${ }^{7}$, mixed dimensions of $\mathrm{vdW}$ integrated electronic and optoelectronic devices ${ }^{2}$. Two-dimensional (2D) layered material building blocks, including graphene, transition metal dichalcogenides (TMDs), hexagonal-boron nitrides, black phosphorus, and Mxene, have recently emerged and increasingly gaining interests for $\mathrm{vdW}$ integration ${ }^{2,6,8}$. The atomically thin, highly crystalline, and dangling bond free basal plane embodies nearly infinite vdW integration platforms for other functional building blocks. The much enhanced $\mathrm{vdW}$ interaction, which scales proportionally with the interacting areas between neighboring objects, enables the creation of a myriad of otherwise impossible artificial heterostructures with new remote epitaxy growth and sometimes unprecedented properties for realizing the non-silicon, atomically thin 2D electronics ${ }^{4,9,10}$.

Beyond these niche demonstrations in 2D/mixed-dimensions and $2 \mathrm{D} / 2 \mathrm{D}$ artificial heterostructures, relatively little is explored toward vertical staking of 3D/3D hetero- (different in chemical compositions) or even homostructures (identical in chemical compositions) that offer a range of technological merits, such as inherent porosity, enhanced surface reactivity and ionic transport, essential for energy harvesting, storage, and catalysis applications. At the root of functional limitation are the intrinsic rigidity and rough surface of $3 \mathrm{D}$ building blocks, preventing the intimate contact within the vdW distance and thus hampering the $\mathrm{vdW}$ interaction. As a consequence, the assembled 3D/3D homo- and heterostructures become irreversibly scrambled upon mechanical agitation or subjecting to external stimuli. We recently demonstrated the spray-drying assembly of the $3 \mathrm{D} / 3 \mathrm{D}$ homostructures comprised of structurally deformed graphene, reduced graphene oxides $(\mathrm{r}-\mathrm{GO})^{11}$, and $\mathrm{MoS}_{2}{ }^{12}$ with intriguing electrochemical and catalytical properties. Still, effective vdW interactions at interfaces remain overshadowed by the inherently rigid nature and the spatially distributed intricate features such as ripples, facets, folds, ridges, and vertices. Moreover, such processes only allow the stacking of 3D/3D homostructures and cannot create complex layouts with well-defined features, periodicities and morphologies as well as the flexibility of stacking directions, and scalability over large areas.

Here, we overcome these limitations of $\mathrm{vdW}$ integration for both 3D/3D homo- and heterostructures by combining the stable dispersion of metallic $1 \mathrm{~T} \mathrm{MoS}_{2}$ polymorph (also termed "chemically exfoliated" $\mathrm{MoS}_{2}$, or ce- $\mathrm{MoS}_{2}$ ) and r-GO inks, the formation of $3 \mathrm{D}$ building blocks, and integration into $3 \mathrm{D} / 3 \mathrm{D}$ homo- and heterostructures in a single step. This is made possible by the use of electrohydrodynamic additive manufacturing (E-3D printing) that exploits a room temperature dewetting process, which we name "dewetting regulated organization of porous structure(s)" (DROPS). The result is the selective patterning of compositionally engineered, mechanically robust, radiation tolerant, and electrochemically active 3D/3D homo- and heterostructures. Specifically, a
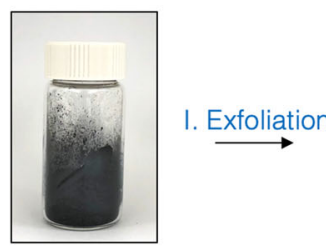

$\mathrm{MoS}_{2}$ powder

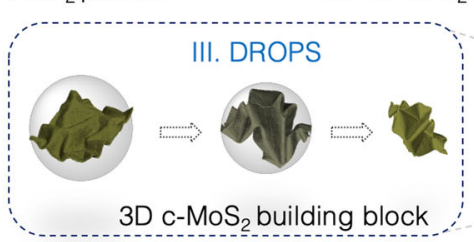

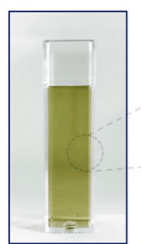

$2 \mathrm{D}$ ce- $\mathrm{MoS}_{2}$ Ink
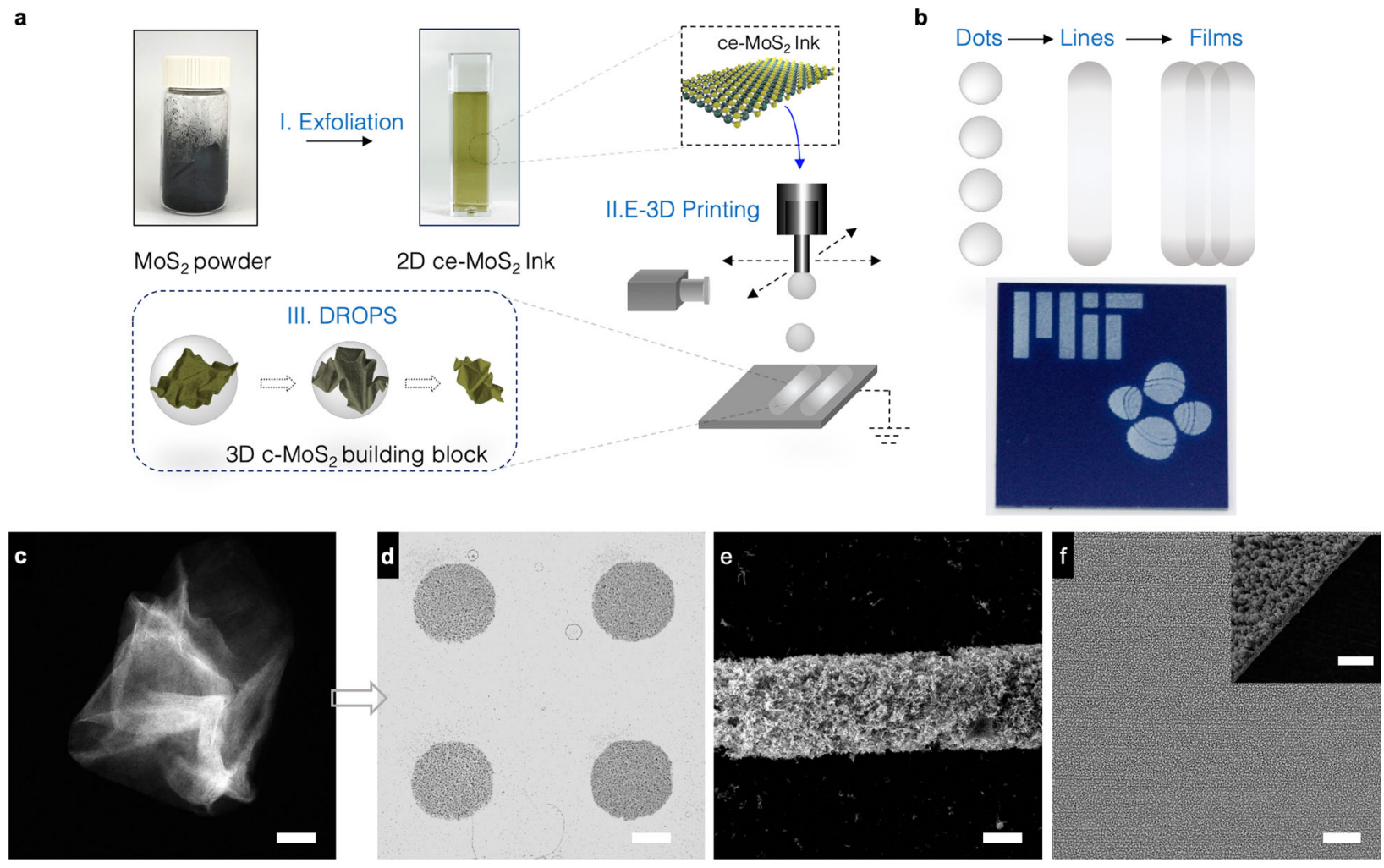

Fig. 1 E-3D printing-DROPS of MoS2 3D/3D homostructures. a Schematic illustration of the E-3D printing-DROPS of MoS $23 D / 3 D$ homostructures shows the process scheme which comprises only three steps: (I) preparation of $2 \mathrm{D}$ ce-MoS $\mathrm{M}_{2}$ inks in aqueous solvents; (II) E-3D printing to create userdefined layouts of 2D ce-MoS 2 inks; and (III) DROPS enables the spontaneous transformation of 2D ce-MoS inks into 3D c-MoS 2 building blocks upon evaporation. b Arbitrary patterns and complex layouts such as KAUST and MIT logos through a computer-controlled X-Y stage that moves the substrates relative to the nozzle. Operation in a raster scanning mode allows circular patterns of droplets to define lines, and lines to merges into films. Dewetting at room temperature results in the crumpling of $2 \mathrm{D}$ ce- $\mathrm{MoS}_{2}$ into 3D nanostructures. c TEM image of the printed 3D c-MoS 2 building blocks. Scale bar is $25 \mathrm{~nm}$. SEM images show an array of $\mathbf{d}$ dots, $\mathbf{e}$ individual line, and $\mathbf{f}$ large area of uniform thin films of MoS $_{2}$ 3D/3D homostructures printed with 3D c-MoS 2 building blocks. Scale bars are $1 \mu \mathrm{m}, 5 \mu \mathrm{m}, 100 \mu \mathrm{m}$, and $10 \mu \mathrm{m}$ (inset), respectively. 


\section{a continuous printing: turbostratic assemblies of 3D/3D homostructures}

\section{b vertical 3D/3D heterostructures}
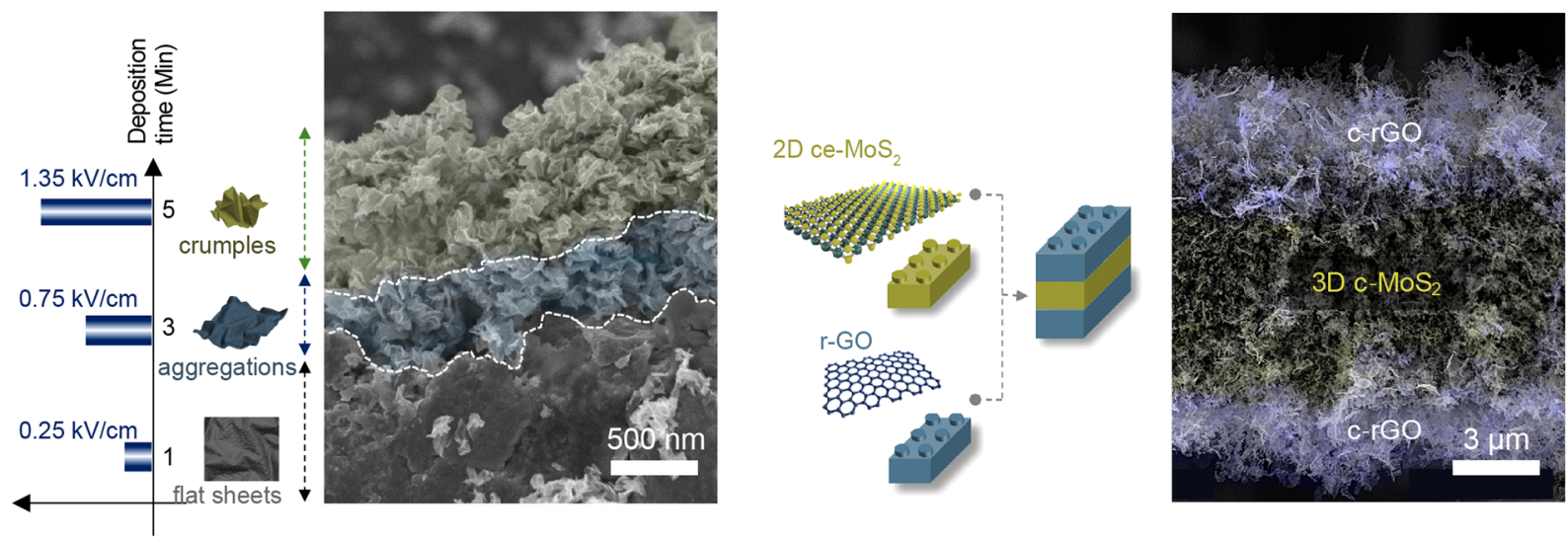

Electric field $(\mathrm{kV} / \mathrm{cm})$

Fig. 2 Design flexibility of E-3D printing-DROPS. a Electric-field vs. deposition-time bar chart diagram demonstrates the capability of creating a single monolith made of the chemically identical compositions but characterized with structurally dissimilar morphologies in a single, continuous deposition. The critical electric field and threshold deposition time of each stage are indicated. Corresponding false-colored, HRSEM image shows the three discrete morphologies, including stacks of flat sheets in gray, heavily wrinkled aggregations in blue, and turbostratic assemblies of crumples in yellow, created through a continuous DROPS deposition coupled with increasing electric field gradients. b Schematic representations feature the direct creation of vertical 3D/3D heterostructures (left). This is accomplished by implementing a dual-sourced DROPS deposition that allows for piecing together two of the most prominent $2 \mathrm{D}$ building blocks along the out-of-plane direction relative to the collecting substrate. Image on the right depicts the corresponding EDS mapping that shows the spatial distribution and uniform coverage of each element (carbon in purple and molybdenum in yellow).

applying electric fields drives the flow of " $2 \mathrm{D}$ inks" from nozzles, followed by the characteristic fission process to achieve droplet sizes as small as $100 \mathrm{~nm}$. An array of multiple nozzles allows rapid and purely additive patterning of 3D/3D homo- and heterostructures with accurate registration. Inks based on either $2 \mathrm{D}$ ce$\mathrm{MoS}_{2}$, rGO or the combination of both can be routinely, reliably, and selectively printed as dots, lines, and films with sub-500-nm dimensions, excellent uniformity and repeatability in thickness (up to few hundred micrometers) in tandem with user-defined layouts, including continuous printing of turbostratic assemblies, vertical, lateral, and even mixed dimensions of $3 \mathrm{D} / 3 \mathrm{D}$ vdW heterostructures, that span length scales from the sub-micrometer to centimeter regimes. These procedures define the location, size, and hierarchical geometry of patterns of 3D/3D vdW heterostructures. High-resolution transmission electron microscopy (HRTEM) observation and spectroscopic characterizations collectively reveal that the $3 \mathrm{D}$ building blocks within $3 \mathrm{D} / 3 \mathrm{D}$ vdW heterostructures are mostly monolayers with spatially distributed wrinkles and ripples. These intricate geometric features are found to first entangle together and then interlocked with each other through elastic buckling upon evaporation, further augmenting the weak vdW integration ${ }^{13}$. To prove the utility, we print out $\mathrm{rGO}-\mathrm{MoS}_{2}$ $3 \mathrm{D} / 3 \mathrm{D}$ vdW heterostructures with a high concentration of metallic $1 \mathrm{~T}$ phase for driving the hydrogen evolution reactions (HER) at extreme $\mathrm{pH}$ values and elevated temperatures.

\section{Results}

It generally requires only three steps to create $3 \mathrm{D} / 3 \mathrm{D}$ vdW homoand heterostructures and does not require a slew of laborious and time-consuming post-engineering processes, thus preserving the atomically clean and electrically addressable interfaces. Figure 1a schematically illustrates the premise of our experimental design that leverages the scalability of synthesizing $2 \mathrm{D}$ ce- $-\mathrm{MoS}_{2}$ inks, and versatility of E-3D printing in terms of printed feature size, geometry, and area coverage in tandem with the utility of DROPS that spontaneously transforming $2 \mathrm{D}$ ce- $\mathrm{MoS}_{2}$ inks to $3 \mathrm{D} \mathrm{MoS}_{2}$ building blocks upon dewetting at room temperatures. $2 \mathrm{D}$ ce- $\mathrm{MoS}_{2}$ inks were prepared through a modified lithium $(\mathrm{Li})$ intercalation and then dispersed in a mixture of deionized water $\left(\mathrm{DI}-\mathrm{H}_{2} \mathrm{O}\right)$ and isopropanol (IPA) with a volume ratio of 1:3. A computerized system of $\mathrm{X}-\mathrm{Y}$ translation stages moves the collecting substrate relative to the spinneret and controls the voltage for printing ce$\mathrm{MoS}_{2}$ containing nanodroplets into various patterns, including circular dots, separated lines, and merged films, in a raster scanning mode. This procedure yields continuous films with predefined micro/nanoscale geometries over macroscopic areas (Fig. 1b) in an automated fashion. Subsequent evaporation of ce- $\mathrm{MoS}_{2}$ containing patterns spontaneously engenders geometric constraints through DROPS, thus affording 3D hierarchically deformed building blocks, namely crumpled $\mathrm{MoS}_{2}\left(\mathrm{c}-\mathrm{MoS}_{2}\right)$. The resulting 3D c-MoS 2 are characterized by particular surface morphologies that foster unconventional structural solutions. These include the introduction of networks of propagated ridges, the formation of jagged facets, the emergence of sharp vertices, shaping corrugated wrinkles, as well as thin and folded edges as shown in HRTEM image (Fig. 1c and Supplementary Scheme 1a). Raman spectra corroborate the HRTEM observation. By tracing the evolution of an in-plane phonon mode at $385 \mathrm{~cm}^{-1}$, assigned to $\mathrm{E}_{2 \mathrm{~g}}^{1}$, of the planar ce- $\mathrm{MoS}_{2}$ sheet, we quantitatively observed a pronounced red shift toward lower wavenumbers, revealing the high strain load of $3 \mathrm{D} / 3 \mathrm{D}$ homostructures (shifted by $1.7 \mathrm{~cm}^{-1}$ per $\%$ strain, $\approx 3.3 \%$, Supplementary Fig. 1). Meanwhile, images of scanning electron microscopy (SEM) demonstrate the formation of 3D/3D c- $\mathrm{MoS}_{2}$ homostructures within the printed circular (Fig. 1d), and linear patterns (Fig. 1e) as well as large area, uniform thin films (Fig. 1f). Energy dispersive X-ray spectroscopy (EDS) mapping of relevant elements, including Mo in green, $S$ in orange, and the overlay of both Mo and S, further demonstrates the generation of printed linear patterns of $3 \mathrm{D} / 3 \mathrm{D}$ vdW homostructures with high aerial densities over a $2 \mathrm{~cm} \times 2 \mathrm{~cm}$ silicon ( $\mathrm{Si}$ ) substrate as shown in Supplementary Fig. 2.

Another disruptive advantage of coupling E-3D printing with DROPS is the greater design freedom. Here, direct printing of $2 \mathrm{D}$ inks into complex 3D layouts with judicious control over 
structural complexity and compositional diversity is readily achieved. As shown in Fig. 2a. chemically identical but structurally dissimilar mixed dimensional $\mathrm{MoS}_{2}$ vdW homostructures can be continuously manufactured through progressively varying the electric field gradients. The resulting mixed dimensional $\mathrm{MoS}_{2}$ $\mathrm{vdW}$ homostructures clearly displayed discrete stages of morphological evolutions upon increasing electric fields. Stacks of flat sheets (gray), heavily wrinkled aggregation (blue), and turbostratic assemblies of crumples (light yellow) can be clearly distinguished in the false-colored, cross-sectional SEM images (Supplementary Fig. 3a, b). The utility of E-3D printing-DROPS approach is further demonstrated by the incorporation of an additional source loaded with $2 \mathrm{D}$ r-GO inks as schematically represented in Fig. $2 \mathrm{~b}$ and Supplementary Fig. 3c. This dualsourced setup closely resembles the thermal evaporation of organic and metallic thin films, where $3 \mathrm{D} / 3 \mathrm{D}$ vdW heterostructures comprised of vertically stacked 3D crumpled r-GO (crGO) and c-MoS 2 building blocks can be pieced together in a layer-by-layer fashion, providing the versatility to control nanoscale features and functionalities on the microscale. Alternatively, both c-rGO and c-MoS $3 \mathrm{D}$ building blocks can be premixed and concurrently deposited to afford 3D/3D heterostructures with compositionally graded lateral regions as elementally mapped in Fig. 3a-d, and Supplementary Fig. 3d. Meanwhile, the flexibility of solution processability also allows us to extend the building block selections from pure $3 \mathrm{D} \mathrm{c}-\mathrm{MoS}_{2}$ and c-rGO building blocks to the combination of both with compositionally engineered ratios (Fig. 3e-i). The ability to sidestep many processing and manufacturing constraints also enables direct deposition of $\mathrm{MoS}_{2} 3 \mathrm{D} / 3 \mathrm{D}$ homostructures onto previously unachievable substrates regardless of textured geometries across various length scales, ranging from micro-textured polystyrene, micro-to-nanostructured carbon clothes (CC) to nanostructured single-walled carbon nanotubes (SWCNTs, Supplementary Scheme 2).

Whereas the E-3D printing defines the printed patterns, periodicity, structural complexity, and compositional diversity, the critical step toward creating 3D/3D homo- or heterostructures hinges strongly on the effectiveness of DROPS. To this end, we verify the requirements on boundary conditions that enables DROPS to transforms 2D ce- $\mathrm{MoS}_{2}$ inks into 3D c- $\mathrm{MoS}_{2}$ building blocks: (1) impinging ce- $\mathrm{MoS}_{2}$ containing medium does not wet the supporting substrate and remains a stable, nonplanar shape upon evaporation, (2) the substrate is chemically nonreactive with the ce- $\mathrm{MoS}_{2}$ containing medium, and (3) printed patterns do not flatten into thin films upon E-3D printing and during evaporation. The first two requirements are satisfied by utilizing low surface energy substrates, such as planar highly oriented pyrolytic graphite (HOPG), silicon/silicon oxides $\left(\mathrm{Si}_{/} / \mathrm{SiO}_{2}\right)$, or highly textured CC. Here, taking HOPG as an example, we compare the interfacial energies between the dispersing medium and HOPG, and derive the first boundary condition through measurable quantities: $\gamma_{12}=\gamma_{13}-\gamma_{23} \cos \theta_{0}>0$, where $\gamma_{12}, \gamma_{13}$, and $\gamma_{23}$ are interfacial energies between dispersing medium/substrate, dispersing substrate/air, and medium/air, $\theta_{0}$ is the equilibrium contact angle of the dispersing medium and supporting substrate, respectively (Supplementary Scheme 3). Next, to ensure the nonplanar dispersing medium is always pinned and stuck on a lateral 3D/3D heterostructures b

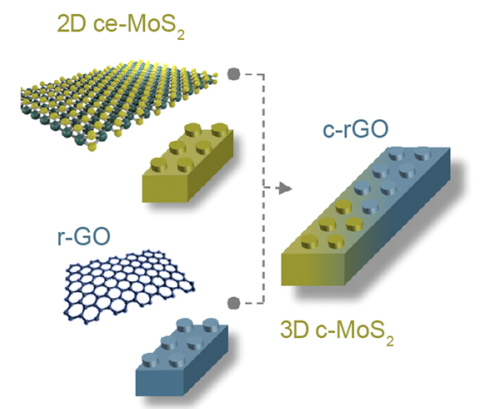

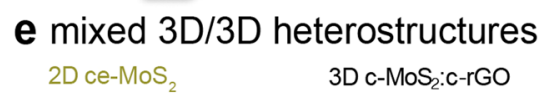
$2: 1$
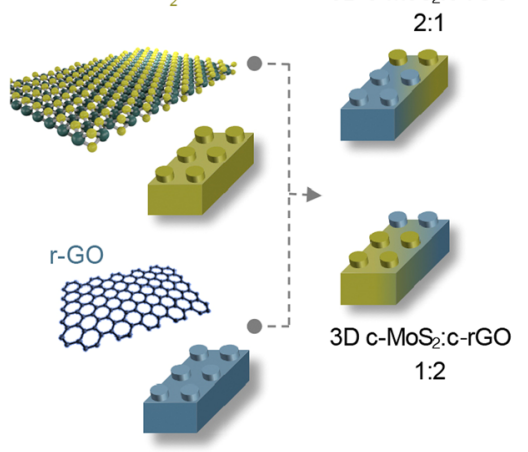

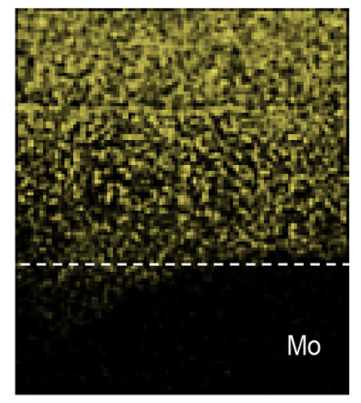

g

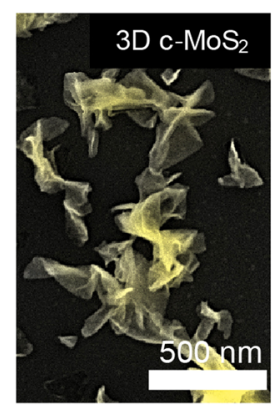

C

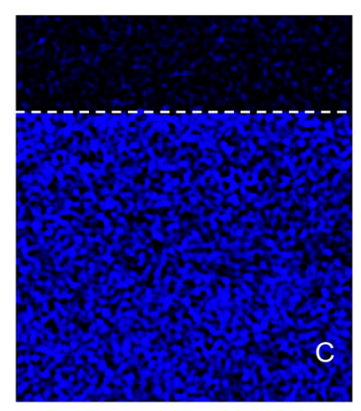

h

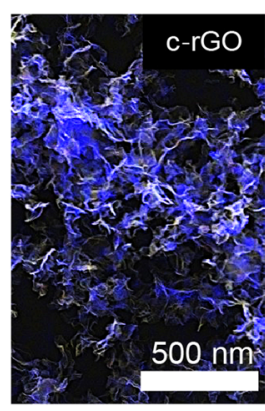

d

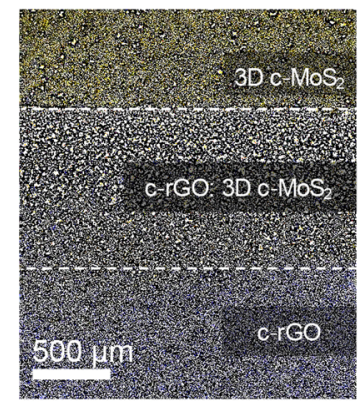

i
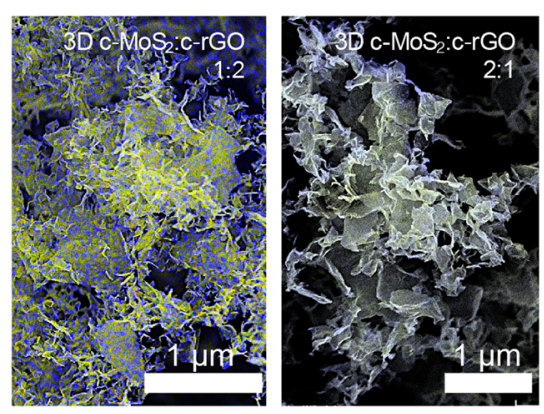

Fig. 3 Utility of E-3D printing-DROPS. a Schematic illustration demonstrates the formation of lateral 3D/3D heterostructures again via the dual-sourced DROPS deposition with controlled depositions of $2 \mathrm{D}$ inks along the in-plane direction. EDS mapping clearly reveals the spatial distribution where individual building blocks on both ends-that is, $\mathbf{b} \mathrm{Mo}$, false-color in yellow, and $\mathbf{c} \mathrm{c}-\mathrm{rGO}$, false-color in blue, gradually converge into lateral heterostructures in the middle (d). In addition, the versatility and flexibility of DROPS allow us to further extend the material selections from pure c-MoS 2 and c-rGO to the combination of both with compositionally engineered ratios as suggested in the scheme (e). 2D inks can be compositionally engineered to create composite inks with desired ratios. Corresponding EDS mappings show the formation of $\mathbf{f}$ pure 3D c-MoS $;$; $\mathbf{g}$ pure c-rGO; $\mathbf{h}$ mixed 3D/3D heterostructure with $\mathrm{c}-\mathrm{MoS}_{2}: \mathrm{c}-\mathrm{rGO}=1: 2$; and $\mathbf{i}$ mixed 3D/3D heterostructure with c-MoS2:c-rGO $=2: 1$, respectively. 
a

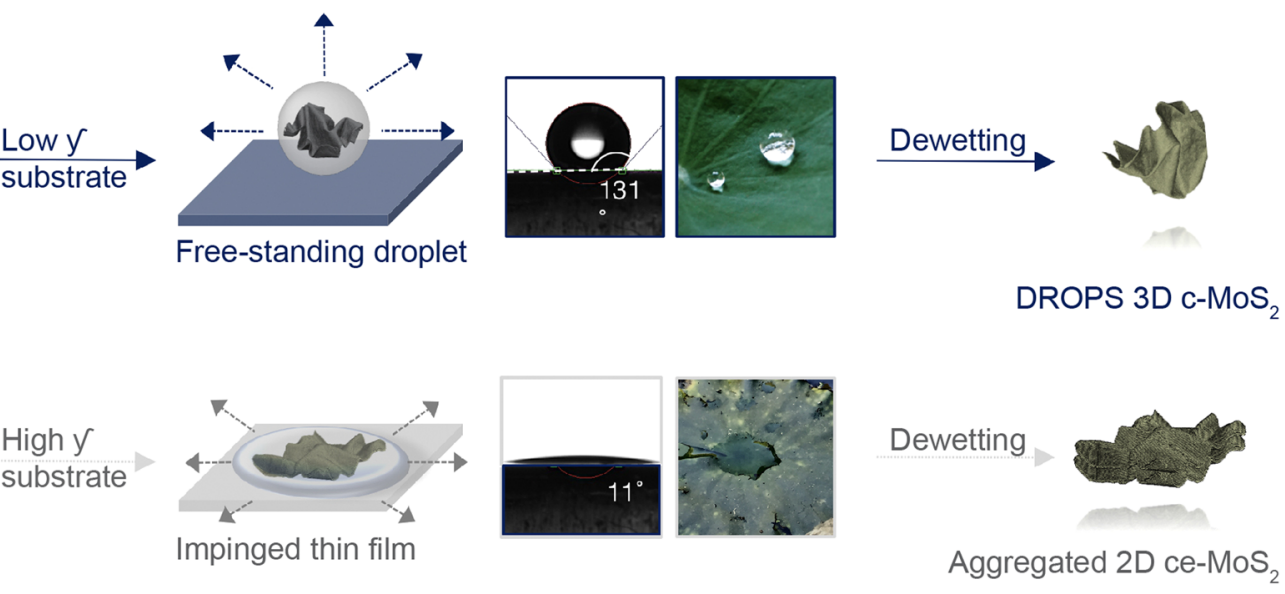

b

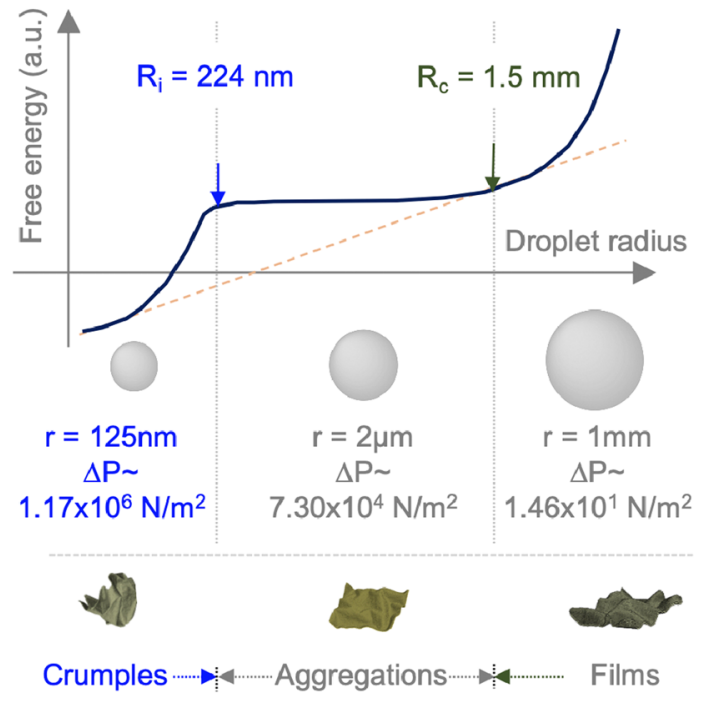

C

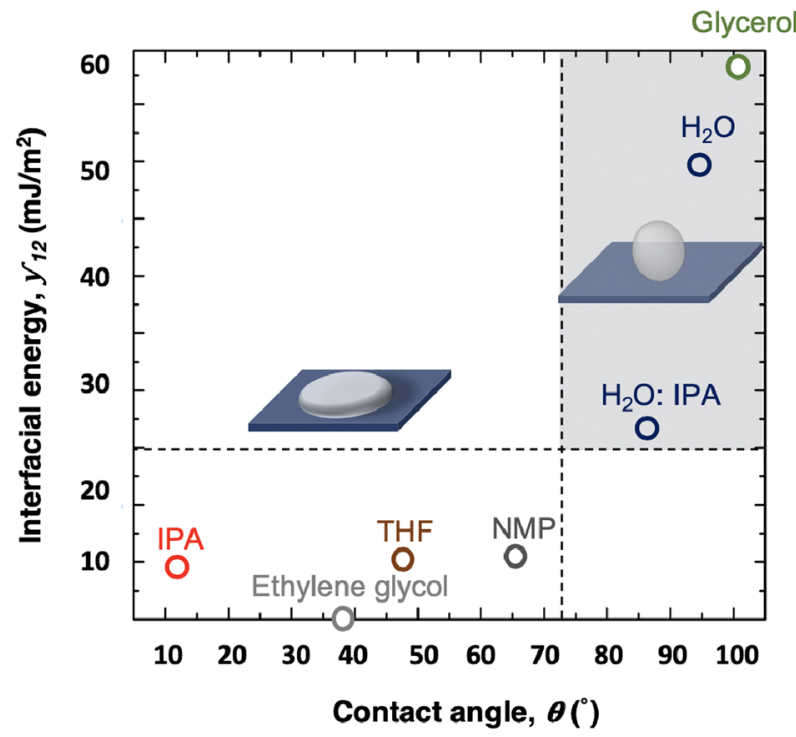

Fig. 4 Mechanistic study of DROPS. a Schematic representation shows distinctively different dewetting dynamics of nano-droplets driven by different interfacial energies at liquid/solid interfaces. $\mathbf{b}$ The diameters of incoming droplets have profound implications on the Laplace pressures $(\Delta P)$ and associated dewetting outcomes. c Contact angles of an array of polar solvents as a function of interfacial energies allow us to deduce the working combinations of the formation of spherical droplets on a HOPG substrate (shaded area, where contact angle $>85^{\circ}$ and interfacial energy $>25 \mathrm{~mJ} / \mathrm{m}^{2}$ ).

HOPG without bouncing, spreading or flattening into thin films, we again deduce the measurable quantities from the manipulation of the work of adhesion between substrate/air/liquid $\left(W_{132}\right)$ which can be expressed by: $W_{132}=W_{12}+W_{33}-W_{13}-W_{23}$, where $W_{132}$ is the work of adhesion at various interfaces, also known as the spreading coefficient and commonly denoted by $S^{14}$

When $W_{132}$ is positive $(S<0)$, dispersing media comprised of $2 \mathrm{D}$ ce- $-\mathrm{MoS}_{2}$ sheets will contract into a nearly spherical shape and pin on HOPG until the completion of evaporation (Fig. 4a). Details of the derivation of working/boundary conditions can be found in Supplementary Discussion, Table 1 and Fig. 4b. In addition, we verify the inflection point radius $\left(R_{i}\right)$ of $244 \mathrm{~nm}$ calculated from $\left(\kappa^{-1} a\right)^{1 / 2}$, where $\kappa^{-1}$ is the capillary length and $a$ is the molecular length of $\mathrm{H}_{2} \mathrm{O}$. It is known that dewetting of droplets with radii below $R_{i}$ does not entail the amplification of longitudinal capillary force that induces flattening into thin films or coalescence of droplets ${ }^{15}$. Only those nano-droplets with radii smaller than $R_{i}$ (collected at a distance of $7.5 \mathrm{~cm}$ away from the tip in the presence of a strong electric field, Supplementary Scheme $2 \mathrm{~b}-\mathrm{f}$ ) remained nearly spherical and pinned on HOPG.
Next, the large overpressure, Laplace pressure, existing in the interior of nano-droplets drives the DROPS at room temperature, spontaneously deforming the $2 \mathrm{D}$ ce- $-\mathrm{MoS}_{2}$ into $3 \mathrm{D} \mathrm{c}-\mathrm{MoS}_{2}$. Based on the Supplementary Discussion, for a nano-droplet of radius $\sim 125 \mathrm{~nm}$, Laplace pressure is typically comparable with an atmospheric pressure of $11.547 \mathrm{~atm}$ or $1.17 \times 10^{6} \mathrm{~N} / \mathrm{m}^{2}$. Importantly, these quantitative descriptors $\left(\gamma_{12}>0, S<0\right.$, and $\left.R<R_{i}\right)$ allow us to forge ahead a range of solvent-substrate combinations for effectively driving DROPS where contact angle $>85^{\circ}$ and interfacial energy $>25 \mathrm{~mJ} / \mathrm{m}^{2}$ (Supplementary Table 1-2 and Fig. 4c). $\mathrm{MoS}_{2} 3 \mathrm{D} / 3 \mathrm{D}$ homostructures can be deposited on a wide variety of soft and rigid substrates while exhibiting a structurally deformed morphology, comparable strain level $(\approx 3 \%)$, and similar dimensions, greatly expanding the options of collecting substrates (Supplementary Table 3).

Conventional manufacturing processes typically reassemble exfoliated $\mathrm{MoS}_{2}$ inks into a continuous but highly aggregated bulk form. Here, soft and adoptable 2D ce- $\mathrm{MoS}_{2}$ inks driven by DROPS first form intimate face-to-face contact at edges with each other and then scroll spatially interconnected struts-like walls within 3D/3D homostructures. This glaring discrepancy in local 
a
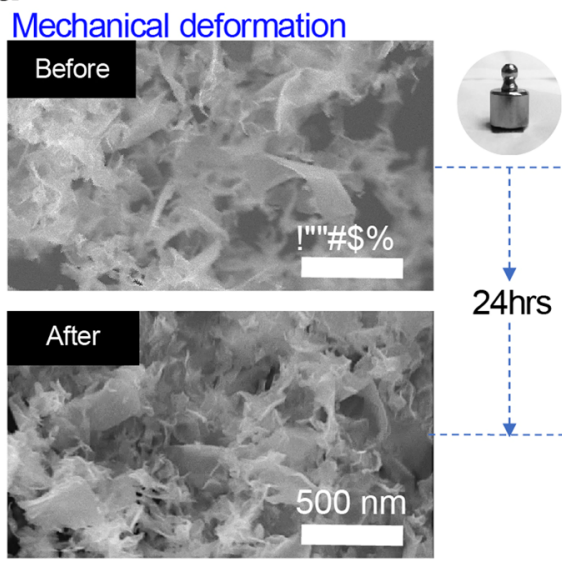

C
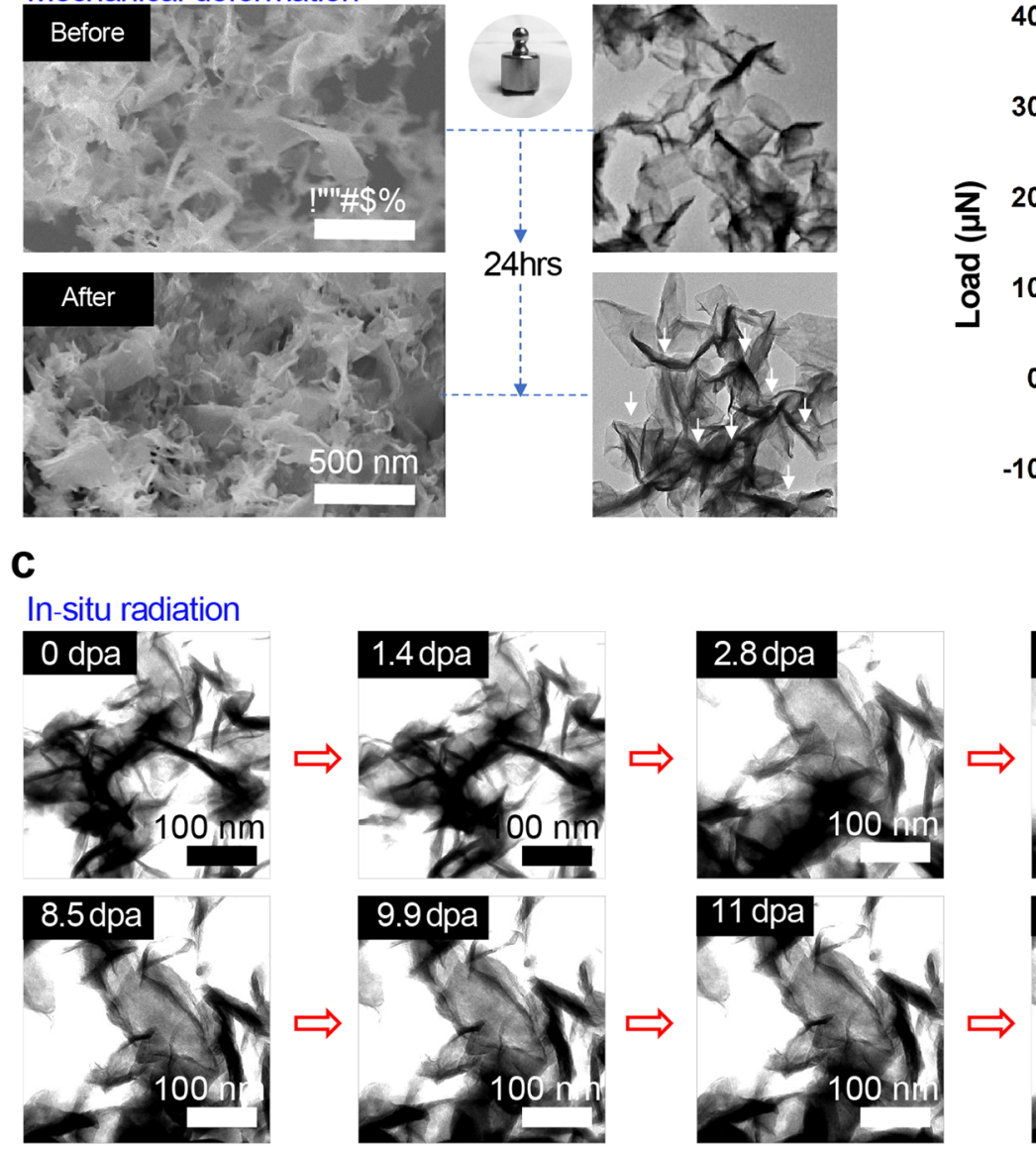

b

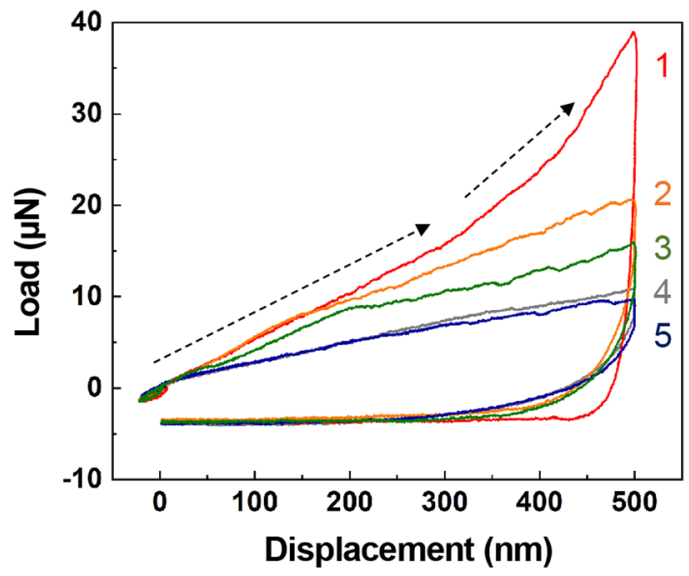

Fig. 5 Mechanically robust, protonically tolerant, and chemically resilient nature of MoS2 3D/3D homostructures. Representative SEM and TEM (inset) images demonstrate the mechanically robust nature of $\mathbf{a} \mathrm{MoS}_{2} 3 \mathrm{D} / 3 \mathrm{D}$ homostructures by virtue of the increasing numbers of elastic buckling of extremely thin edges (arrows in white color) after subjected to an external load for $24 \mathrm{~h}$. $\mathbf{b}$ Load/displacement curves of five cycles of compression and release on $\mathrm{MoS}_{2}$ 3D/3D homostructures show the initially elastic response to displacement of $300 \mathrm{~nm}$ at an external load of $17 \mu \mathrm{N}$. c A series of still frames derived from continuous TEM imaging under an in-situ $46 \mathrm{keV} \mathrm{Au}{ }^{+}$reveals the high radiation tolerance of $\mathrm{MoS}_{2} 3 \mathrm{D} / 3 \mathrm{D}$ homostructures.

structure rearrangements at nanoscale has important implications in the overall packing and thus the resistance to external loads. Similar to conventional rGO or $\mathrm{MoS}_{2} 3 \mathrm{D} / 3 \mathrm{D}$ homostructures held by weak vdW interactions, the disruptive packing of $3 \mathrm{D} \mathrm{c}-$ $\mathrm{MoS}_{2}$ is manifested in the broadening XRD patterns and the absence of characteristic (002) peak of planar 2D ce- $\mathrm{MoS}_{2}$ counterparts assigned at $16.39^{\circ}$ (Supplementary Fig. 4). Meanwhile, networks of spatially interconnected struts within the $3 \mathrm{D} /$ 3D homostructures were found to withstand external loads without incurring catastrophic collapse. As shown in Fig. 5a, the structural integrity of $\mathrm{MoS}_{2} 3 \mathrm{D} / 3 \mathrm{D}$ vdW homostructures with a thickness of $10 \mu \mathrm{m}$ remains intact after deformation for a duration of $24 \mathrm{~h}$. This can be explained by the merging, scrolling, and elastic buckling of extremely thin and flexible edges from neighboring 3D c- $\mathrm{MoS}_{2}$ that forms resilient struts (HRTEM, right panel of Fig. 5a and Supplementary Scheme 1b, c) ${ }^{16,17}$. As indicated in Fig. 5b, load-displacement curves further reveal two regimes of deformation: (a) nearly linear elastic regime, corresponding to elastic buckling of scrolling edges and struts; and (b) abrupt load increasing regime due to the densification of crumples that ultimately become stiffer by forming myriad hard-tobend ridges. In contrast, $\mathrm{MoS}_{2}$ 3D/3D homostructures prepared from an aerosol assembly were found to irreversibly pelletized into large, irregular grains by virtue of the strain-hardening effect on the relatively thick walls during compression (Supplementary Fig. 5) ${ }^{18}$.

In parallel, in-situ ion irradiation TEM ( ${ }^{3}$ TEM) of $46 \mathrm{keV} \mathrm{Au}{ }^{+}$ bombardment during nanoscale observation revealed a high tolerance of radiation doses of $\mathrm{MoS}_{2}$ 3D/3D homostructures ${ }^{19}$. The printed $\mathrm{MoS}_{2} 3 \mathrm{D} / 3 \mathrm{D}$ homostructures samples were irradiated to a fluence of $3 \times 10^{15}$ ions $/ \mathrm{cm}^{2}$ for $105 \mathrm{~min}$ through the use of a defocused $46 \mathrm{keV} \mathrm{Au}{ }^{+}$ion beam at nominally room temperature to impart the greatest amount of damage as seen in 30 displacement per atoms (dpa). This in-situ irradiation was conducted while using traditional bright-field TEM imaging modes, allowing us to observe the structural evolution in-situ with increasing irradiation duration. As featured in Fig. $5 c$, a series of still frames from video collected in-situ during $\mathrm{Au}^{+}$irradiation suggests that continuous ion irradiation did not result in significant structural degradation or distortion, and each $3 \mathrm{D} c-\mathrm{MoS}_{2}$ building blocks remain tightly bound at edges. The ability to withstand such an irradiation environment can directly be translated to their response when exposed to space environments ${ }^{20}$. Together with the high refractive index $(>4)$, moderately high absorption, and moderate mass density, the prospect of additively manufacturing of mechanically robust and radiation tolerant $\mathrm{MoS}_{2}$ 3D/3D homostructures embodies an exciting direction which is likely to contribute a wealth of revealing 

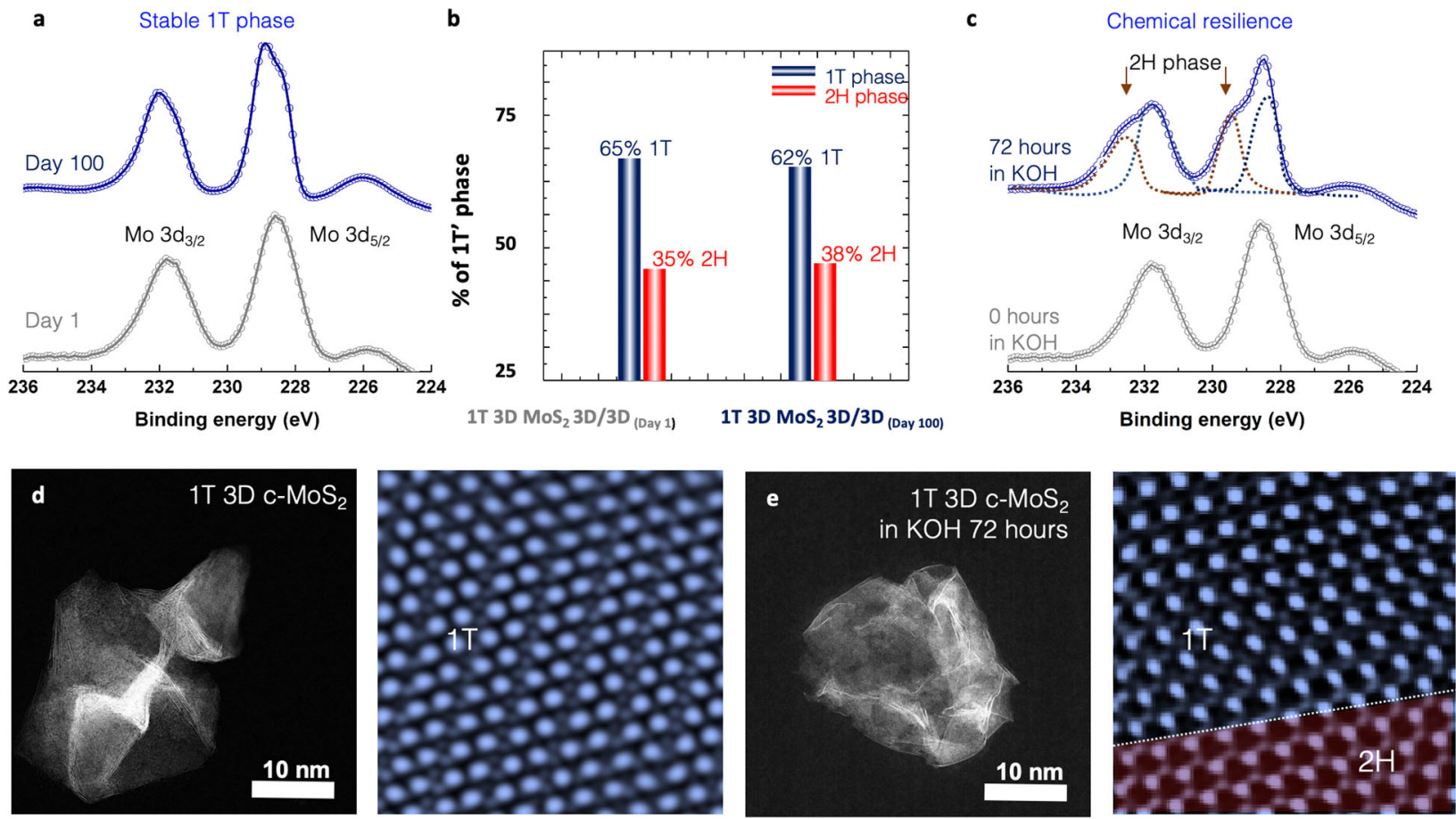

Fig. 6 Chemical resilience of MoS2 3D/3D homostructures. a, b By tracing the evolution of $1 \mathrm{~T}^{\prime}$ Mo $3 \mathrm{~d}$ core level photoemission peaks at $228.1 \mathrm{eV}$ (Mo

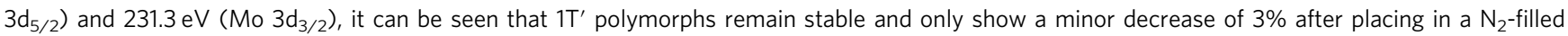
glovebox for 100 days. c XPS characterization, d, e TEM images along with false-colored HAADF analysis (1T' clustered Mo in blue and 2H trigonal prismatic Mo in red), collectively attest to the premise that intricate morphological features help to preserve the conductive $1 \mathrm{~T}^{\prime}$ phase after continuous electrochemical cycling in $1 \mathrm{M} \mathrm{KOH}$ for $72 \mathrm{~h}$.

insights toward the practical design of new structural composites for spacecraft as well as active electrodes for catalysis and lightweight, compact energy storage applications to propel future space missions beyond our solar system ${ }^{21}$.

Another distinctive advantage of room temperature E-3D printing-DROPS is the concurrent retention of an otherwise impossible high concentration of $1 \mathrm{~T}$ metallic phase throughout the course of manufacturing. Phase engineering plays an important role in transport across atomically sharp interfaces and has profound implications on properties such as conductivity, catalytic reactivity, and chemical stability ${ }^{22}$. Unfortunately, the advantageous feat of phase engineering tends to fade quickly due to the metastable and temperature vulnerable nature of $1 \mathrm{~T}$ phase. Here, combining additive manufacturing, vdW integration and self-crumpling of $2 \mathrm{D}$ ce- $\mathrm{MoS}_{2}$ in a single E-3D printing-DROPS step offers new promises to overcome these phase engineering constraints. As confirmed in Fig. $5 \mathrm{~g}$ and Supplementary Scheme $1 \mathrm{~d}, 1 \mathrm{~T}$ phase can be atomically resolved through the aberrationcorrected scanning transmission electron microscopy (STEM) operating at high-angle annular dark field (HAADF) mode. Meanwhile, the chemical states of Mo were investigated by X-ray photoelectron spectroscopy (XPS) and Raman spectroscopy on thin films of $\mathrm{MoS}_{2}$ 3D/3D homostructures as suggested in Fig. $6 \mathrm{a}$. Specifically, deconvolution of Mo $3 \mathrm{~d}$ core level region showed two signature peaks at $228.1 \mathrm{eV}\left(\right.$ Mo $\left.3 \mathrm{~d}_{5 / 2}\right)$ and $231.3 \mathrm{eV}\left(\right.$ Mo $\left.3 \mathrm{~d}_{3 / 2}\right)$, characteristic of $1 \mathrm{~T}$ phase, relative to the $2 \mathrm{H} \mathrm{MoS}$ standard

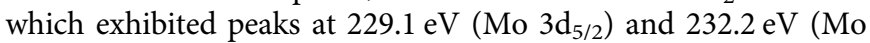
$3 \mathrm{~d}_{3 / 2}$ ). Integration of areas under these core level photoemission peaks reveals a predominant $1 \mathrm{~T}$ phase fraction of $65 \%$ for $\mathrm{MoS}_{2}$ $3 \mathrm{D} / 3 \mathrm{D}$ homostructures and remains substantially stable even after 100 days under ambient conditions (Fig. 6b). The retention of a high percentage of $1 \mathrm{~T}$ metallic phase is also evident on the much-enhanced electrical conductivity. Indeed, Supplementary Fig. 6 provides a photograph of our design for resistance measurement of $1 \mathrm{~T} \mathrm{MoS}_{2} 3 \mathrm{D} / 3 \mathrm{D}$ homostructures. The thicknessnormalized electrical conductivity of $\mathrm{MoS}_{2}$ 3D/3D homostructures is determined to be $34 \pm 6 \mathrm{~S} \mathrm{~cm}^{-1}$ while the stacked 2D/2D sheets deliver a slightly higher electrical conductivity of $55 \pm 4 \mathrm{~S} \mathrm{~cm}^{-1}$.

In addition to preserved $1 \mathrm{~T}$ phase stability at ambient conditions, combined XPS, HRTEM, and HAADF-STEM studies collectively demonstrated the chemical resilience of the $1 \mathrm{~T}$ phase of $\mathrm{MoS}_{2}$ 3D/3D homostructures even after continuous immersing in $1 \mathrm{M} \mathrm{KOH}$ for $72 \mathrm{~h}$. We did not observe noticeable changes in the overall structure which remained solid except for the emergence of many corrugated ridges (Fig. 6c-e). Meanwhile, HAADFSTEM analysis revealed that a decreased concentration of strained $1 \mathrm{~T}$ regions (octahedral, false-colored as blue). Gradual relaxation of Mo atoms from $1 \mathrm{~T}$ lines to symmetrical $2 \mathrm{H}$ spacing (trigonal prismatic, false-colored as red) can be clearly identified, corroborating the results collected from the time-dependent XPS (Fig. 6b). By tracing the time-dependent variation of $1 \mathrm{~T}$ phase concentrations of $\mathrm{MoS}_{2}$ 3D/3D homostructures in $1 \mathrm{M} \mathrm{KOH}$ (Fig. 6b, and Supplementary Fig. 7a, b), it becomes clear that the $2 \mathrm{H}$ phase begins to take hold after $12 \mathrm{~h}$ of cycling tests, reaches its peak after $24 \mathrm{~h}$, and ultimately saturates after $72 \mathrm{~h}$ of continuous operation. The saturation of $2 \mathrm{H}$ phase concentration may be the result of the entanglement of dislocations. Propagating strain fields radiated form these dislocations form an additional energetic barrier to hinder the $1 \mathrm{~T}$-to- $2 \mathrm{H}$ phase transformation. By comparison of planar $1 \mathrm{~T} 2 \mathrm{D}$ ce- $-\mathrm{MoS}_{2}$, the morphology did not alter significantly except for the increased numbers of tears and pinholes as examined by TEM (Supplementary Fig. 8a, b). Yet, false colored HAADF-STEM analysis confirms the complete phase transformation after $72 \mathrm{~h}$ of operation in $1 \mathrm{M} \mathrm{KOH}$, meshing well with the XPS as shown in Supplementary Fig. 8c. Deconvolution of Mo $3 \mathrm{~d}$ core level region along with integrated peak area ratios from planar $1 \mathrm{~T}$ ce- $-\mathrm{MoS}_{2}$ shows that substantial 

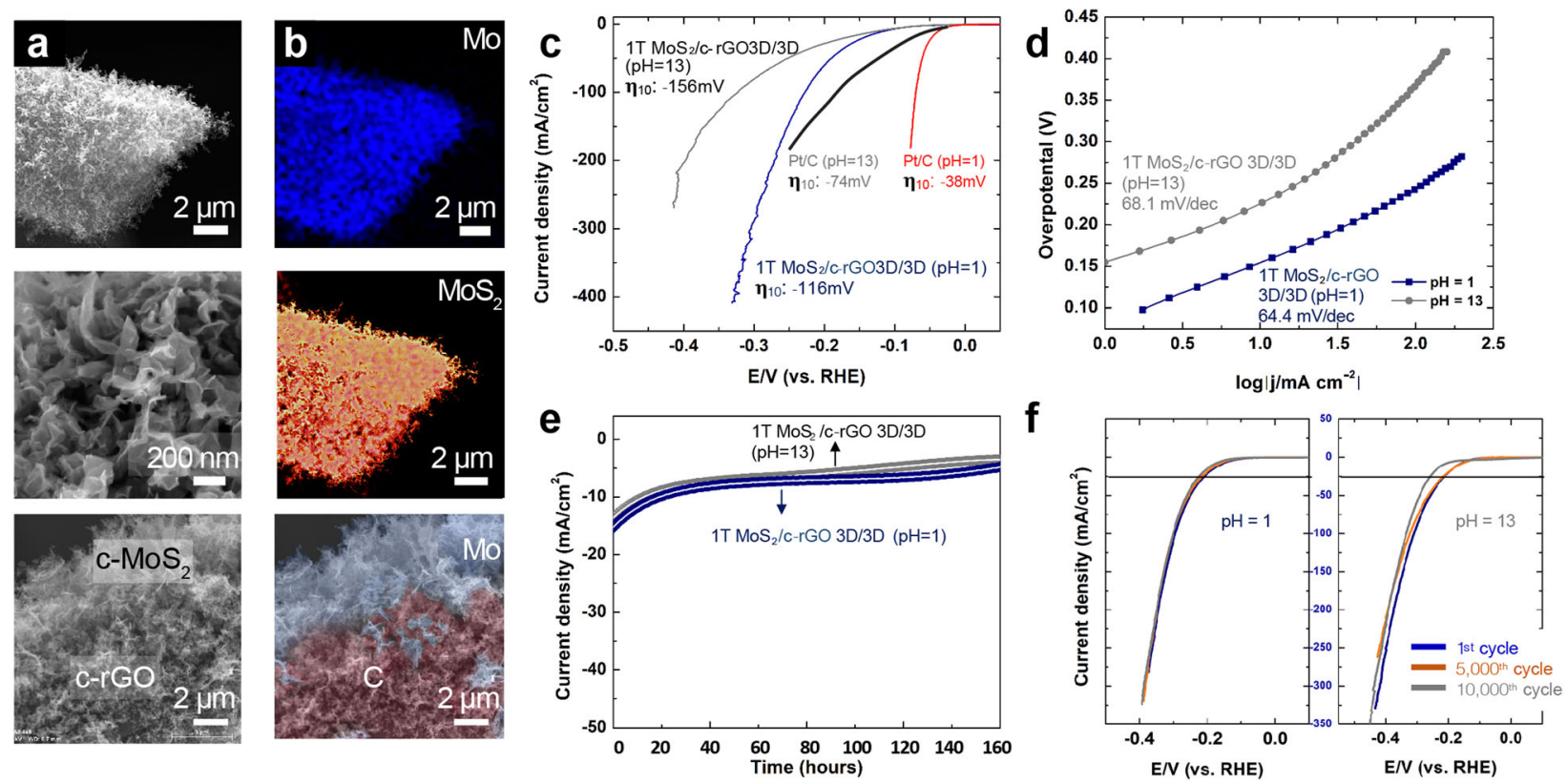

Fig. 7 Comparison of HER metrics in both 0.5 M H2SO4 and $1 \mathbf{M ~ K O H . ~ a ~ S E M ~ i m a g e s ~ t a k e n ~ a t ~ d i f f e r e n t ~ p e r s p e c t i v e s , ~ i n c l u d i n g ~ ( t o p ) ~ t i l t e d - , ~ ( m i d d l e ) ~ t o p - , ~}$ show a highly uniform and dense coverage of $1 \mathrm{~T}^{\prime} \mathrm{MoS}_{2} / \mathrm{c}-\mathrm{rGO} 3 \mathrm{D} / 3 \mathrm{D}$ heterostructures supported on CC. (Bottom) A cross-sectional view of an area where top layers of $\mathrm{c}-\mathrm{MoS}_{2}$ were removed, reveals the formation of vertical 3D/3D heterostructures. $\mathbf{b}$ Corresponding EDS mapping shows the chemical composition and spatial distribution of each element: $\mathrm{Mo}$ in blue, $\mathrm{C}$ in red, and an overlap of both $\mathrm{Mo}$ and $\mathrm{S}$, respectively. HER metrics extrapolated from $\mathbf{c}$ LSV curves, and $\mathbf{d}$ Tafel slopes of $1 \mathrm{~T} \mathrm{MoS} / \mathrm{c}-\mathrm{rGO} 3 \mathrm{D} / 3 \mathrm{D}$ heterostructures display comparable performance in both $0.5 \mathrm{M} \mathrm{H}_{2} \mathrm{SO}_{4}$ (line in naval blue) and 1 $\mathrm{M} \mathrm{KOH}$ (line in gray). In contrast, $\mathrm{Pt} / \mathrm{C}$ degrades significantly in $1 \mathrm{M} \mathrm{KOH}$. e Time-dependent current density curves of HER electrodes made of $1 \mathrm{~T}^{\prime} \mathrm{MoS}_{2} /$ c-rGO 3D/3D heterostructures taken under respective $\eta_{10}$ for $180 \mathrm{~h}$ together with $\mathbf{f} \mathrm{LSV}$ curves after continuous potential sweeps at $5 \mathrm{mV} \mathrm{s} \mathrm{s}^{-1}$ in $0.5 \mathrm{M}$ $\mathrm{H}_{2} \mathrm{SO}_{4}$ (left), and $1 \mathrm{M} \mathrm{KOH}$ (right) after 1st, 5000th, and 10,000th potential sweeps show electrochemically stable output even after prolonged operation.

phase reversion occurred shortly after $6 \mathrm{~h}$ of electrochemical cycling (a $2 \mathrm{H}$ phase fraction of $\sim 60 \%$ ) and the remaining $1 \mathrm{~T}$ phase completely reverted back to $2 \mathrm{H}$ phase after $12 \mathrm{~h}$ of operation.

The E-3D printing-DROPS approach enables the direct and scalable formation of 3D/3D homo- and heterostructures with a unique combination of high porosity, high radiation tolerance, and structural integrity, chemically stable $1 \mathrm{~T}$ phase, and enhanced electrical conductivity, opening up new avenues for a wide range of applications (Supplementary Scheme 4). As a proof of concept, we demonstrated the direct printing of mix dimensional $\mathrm{MoS}_{2}$-rGO 3D/3D heterostructures as the electrode for driving $\mathrm{pH}$ universal HER, which is one of the key processing steps in production of carbon-neutral chemical fuels ${ }^{23-25}$. Despite extensive efforts on the exploration of new catalysts, platinum $(\mathrm{Pt})$ remains the current benchmark for $\mathrm{HER}^{26}$. By using E-3D printing-DROPS, large area and uniform $3 \mathrm{D} / 3 \mathrm{D}$ $\mathrm{MoS}_{2} / \mathrm{c}$-rGO heterostructures can be directly printed out on micro/nanotextured and fibrous CC substrates as HER catalysts (areal mass loading of $13 \mu \mathrm{g} / \mathrm{cm}^{2}$ and active area of $1 \mathrm{~cm} \times 1 \mathrm{~cm}$, Fig. 7a). In this design, we introduced c-rGO to improve the overall electrical connection between the $\mathrm{MoS}_{2}$ catalysts and underlying CC substrates.

Cross-sectional EDS mapping of relevant elements, including Mo in blue, $\mathrm{C}$ in red, and the overlay of both Mo and C, closely resembles the bilayers of spatially percolating networks of $\mathrm{MoS}_{2} /$ c-rGO 3D/3D heterostructures as demonstrated in Fig. $7 \mathrm{~b}$. Despite deceptively simple in its processing, E-3D printingDROPS induced nanostructuring is indeed very rich in electrochemical and catalytic insights as concluded in Supplementary Scheme 4 . In addition to the activated basal plane with an optimized Gibbs free energy, $\Delta G_{H}$ via strained-S vacancies and high electrochemically active surface area (EASA) enabled by the $3 \mathrm{D}$ multiscale porous morphology, the environmentally benign and synthetically scalable dewetting process, which works at room temperature largely preserves the metastable, crystallographically distorted 1T phase, further facilitating electronic connections between active sites and underlying current collector.

We successfully catalyze efficient HER at extreme $\mathrm{pH}$ values $(\mathrm{pH}$ 1 vs. $\mathrm{pH} 13$ ) and elevated temperatures (up to $300^{\circ} \mathrm{C}$ ) up to 10,000 cycles. In addition to delivering the much enhanced HER activities in acid $\left(\eta_{10}\right.$, overpotential, of $-0.116 \mathrm{~V}$, and TOF, turnover frequency, of $0.577 \mathrm{~s}^{-1}$ ), the printed $\mathrm{MoS}_{2} / \mathrm{c}-\mathrm{rGO} 3 \mathrm{D} / 3 \mathrm{D}$ heterostructures exhibited high total electrode and intrinsic HER activities in basic, with a $\eta_{10}$ of $-0.154 \mathrm{~V}$, a Tafel slope of $68.1 \mathrm{mV} / \mathrm{dec}$, an EASA of $601.66 \mathrm{~cm}^{2}$, high current densities of $-250 \mathrm{~mA} / \mathrm{cm}^{2}$ at less than $-0.4 \mathrm{~V}$, and a TOF of $0.311 \mathrm{H}_{2}$ molecules per second (Fig. $7 \mathrm{c}$ and d, Supplementary Fig. 9, and Supplementary Table 4). As a comparison, commercial $\mathrm{Pt} / \mathrm{C}$ catalysts (40\% Pt supported on carbon black with mass loading of $0.8 \mathrm{mg} / \mathrm{cm}^{2}$ ) were included. Combined theoretical studies and experimental observations have collectively shown that sluggish water adsorption and dissociation dynamics have adversely affected HER in base electrolytes ${ }^{27-32}$. Here, it can be seen that the Tafel slopes of $1 \mathrm{~T} \mathrm{MoS}_{2} / \mathrm{c}-\mathrm{rGO} 3 \mathrm{D} / 3 \mathrm{D}$ heterostructures (Fig. $7 \mathrm{~d}$ ) attained in a low $\mathrm{pH}$ condition remained reasonably low $(64.4 \mathrm{mV} / \mathrm{dec})$ and did not increase much when measured in a high $\mathrm{pH}$ condition $(68.1 \mathrm{mV} / \mathrm{dec})$, suggesting an efficient Heyrovsky mechanism at the activated basal plane and edges of $1 \mathrm{~T} 3 \mathrm{D} c-\mathrm{MoS}_{2}$ with the hydrogen desorption reaction as the limiting step: $\mathrm{H}_{\text {ads }}+\mathrm{H}_{3} \mathrm{O}^{+}+\mathrm{e}^{-} \rightarrow \mathrm{H}_{2}+\mathrm{H}_{2} \mathrm{O}$ (in an acidic condition) and $\mathrm{H}_{\text {ads }}+\mathrm{H}_{2} \mathrm{O}+\mathrm{e}^{-} \rightarrow \mathrm{OH}^{-}+\mathrm{H}_{2}$ (in a basic condition), respectively.

Detailed discussion of $\mathrm{pH}$ resistant phase, electronic connections and mechanisms can be found in the Supplementary 
Discussion. Meanwhile, Tafel slopes of Pt/C degrades significantly in basic electrolytes $(30.3 \mathrm{mV} / \mathrm{dec}$ in $\mathrm{pH} 1 \mathrm{vs.} 113 \mathrm{mV} / \mathrm{dec}, \mathrm{pH} 13$, Supplementary Fig. 9). Electrochemical impedance spectroscopy (EIS, Supplementary Fig. 10) further indicates the formation of electrically addressable and $\mathrm{pH}$ resistant connections across strained-S vacancy sites, conductive c-rGO and current collecting CC substrates, leading to efficient transport across the electrode/ electrolyte interfaces with the significantly reduced charge transfer resistance $\left(R_{c t}, 1.5 \Omega\right.$ of $\mathrm{pH} 1$ vs. $4.1 \Omega$ of $\left.\mathrm{pH} 13\right)$. Yet, series resistance $\left(R_{s}\right)$ measured in $1 \mathrm{M} \mathrm{KOH}$ did not change much relative to the $R_{s}$ obtained in $0.5 \mathrm{M} \mathrm{H}_{2} \mathrm{SO}_{4}$. $(1.2 \Omega$ in pH 13 vs. 1.1 $\Omega$ in $\mathrm{pH}$ 1). The $\mathrm{pH}$ resistant electronic connections established by the rich $1 \mathrm{~T}$ phase propagating along the $3 \mathrm{D}$ porous morphology also manifest in the low $\eta_{10}$ of $-0.25 \mathrm{~V}$ in an acidic medium and $-0.37 \mathrm{~V}$ in a basic medium at a current density of $-200 \mathrm{~mA} / \mathrm{cm}^{2}$, small Tafel slopes of $72 \mathrm{mV} / \mathrm{dec}$ (in acid) and $77.4 \mathrm{mV} / \mathrm{dec}$ (in base). We also performed prolonged electrolysis at constant potential $(-116 \mathrm{mV}$ in $\mathrm{pH} 1$ vs. $-154 \mathrm{mV}$ in $\mathrm{pH} 13)$ to study HER performance under continuous operation and observed a negligible change after $160 \mathrm{~h}$ of continuous operation, as suggested in Fig. 7e.

In line with electrochemical stability tests, the HER catalytic activity showed a minor shift of $\sim 10 \mathrm{mV}$ with respective to $\eta_{10}$ after undergoing 5000 sweeps, and then appear rather stable after 10,000 sweeps in an acidic environment (Fig. 7f). On the other hand, a moderate loss of overpotentials by $\sim 5 \%$ was observed at the end of LSV sweeps $\left(10,000\right.$ sweeps) in the basic electrolyte ${ }^{33}$. More significantly, the $\mathrm{MoS}_{2} / \mathrm{c}-\mathrm{rGO} 3 \mathrm{D} / 3 \mathrm{D}$ heterostructures remains catalytically functional in both low and high $\mathrm{pH}$ conditions under continuous operation of 10,000 cycles or after annealing at $300{ }^{\circ} \mathrm{C}$ (Supplementary Fig. $11-16$ and see Supplementary Discussion for thermally resistant HER). $2 \mathrm{H} \mathrm{MoS} / \mathrm{c}$ rGO 3D/3D heterostructures based HER electrode retained exceptionally high current densities $\left(\sim 385 \mathrm{~mA} / \mathrm{cm}^{2}\right.$ at $-0.36 \mathrm{~V}$ in pH 1 vs. $\sim 255 \mathrm{~mA} / \mathrm{cm}^{2}$ at $-0.42 \mathrm{~V}$ in pH 13) while $\eta_{10}, R_{s}$, and Tafel slopes only increased slightly relative to those of $1 \mathrm{~T} \mathrm{MoS} / \mathrm{c}$ rGO 3D/3D heterostructures ${ }^{34-39}$. The HER performance at each active site may be further enhanced through introducing strategies, such as morphology and surface chemistry engineering ${ }^{26}$, chemical tethering to induce orbital modulation 36,40 , atomic engineering of metal doping sites ${ }^{41}$, to ultimately surpass the current benchmark, Pt-based HER catalysts, as well as those of metal carbide doped $\mathrm{TMDs}^{26}$, selenide-based, and non-noble metal-based electrocatalysts in base (see Supplementary Table 46 for a detailed literature comparison $)^{26,40-42}$ for industrial scale implementation.

\section{Discussion}

These pilot demonstrations of printable $3 \mathrm{D} / 3 \mathrm{D}$ vdW homo- and heterostructures embody many exciting inroads in the construction of a new class of electronic, optoelectronic, or magnetic devices with the unprecedented performance or entirely new functions beyond the reach of the existing materials. Yet, we are only scratching the surface of what is possible. With the rapidly expanding frontiers of $2 \mathrm{D}$ and layered materials, a much broader range of $2 \mathrm{D}$ layered material inks can be isolated, mixed, matched, and printed to create a whole gamut of complex architectures with tailored functionalities and improved material properties through this E-3D printing-DROPS strategy ${ }^{43}$. More importantly, the prospect of making combinatorial libraries of $\mathrm{vdW} 3 \mathrm{D} / 3 \mathrm{D}$ heterostructures represents an exciting new direction that will likely contribute a wealth of revealing insights capable of meeting emerging needs in energy storage, harvesting and conversion, catalysis and sensing, and many more areas that are beyond the reach of current technologies.

\section{Methods}

General description of the E-3D printing-DROPS strategy. Experiments were performed using a customized E-3D printing setup as shown in Fig. 1a. All HER electrodes were prepared via the E-3D printing-DROPS strategy at room temperature and no post-annealing was performed. Prior to deposition, all substrates, including $\mathrm{HOPG}, \mathrm{CC}$, glassy carbon, $\mathrm{Si} / \mathrm{SiO}_{2}$, ITO, and SWCNT thin films, were cleaned through UV/ozone treatment for $20 \mathrm{~min}$. To prepare $1 \mathrm{~T} \mathrm{MoS}_{2} / \mathrm{c}-\mathrm{rGO} 3 \mathrm{D} /$ $3 \mathrm{D}$ heterostructures with the highest strain loads $(\sim 3.3 \%)$, a mixture of DI- $\mathrm{H}_{2} \mathrm{O}$ and IPA (volume ratio of $1: 3, \mathrm{v} / \mathrm{v}$ ) was used as the solvent to impart fast evaporation. Next, aqueous solutions of ce- $\mathrm{MoS}_{2}$ inks $(250 \mu \mathrm{g} / \mathrm{mL})$ were fed to the spinneret (gauge $18 \mathrm{TW}$ needle) by a programmable syringe pump. An external electric field of $1.35 \mathrm{kV} / \mathrm{cm}$ was generated with a high-power supply (ES 40P-20 W/ DAM, Gamma high voltage research). Computerized multi-pass deposition was achieved through the integration of an $x-y$ translational stage (LTS 300, Thorlabs) at various linear stage speeds up to $2.5 \mathrm{~mm} / \mathrm{s}$. The flow rate was carefully maintained at $5 \mu \mathrm{L} / \mathrm{min}$ to achieve the high yield of $1 \mathrm{~T} 3 \mathrm{D} c-\mathrm{MoS}_{2}$. With matching surface chemistry and boundary conditions, droplets remained nearly spherical after deposition and then spontaneously dewet to drive the transformation of flat ce- $\mathrm{MoS}_{2}$ sheets into porous yet interconnected $\mathrm{MoS}_{2}$ 3D/3D nanostructures. A high-speed camera was implemented to observe and adjust the flow rate in a timely fashion, thus suppressing the unwanted aggregation of ce- $\mathrm{MoS}_{2}$ sheets within the printed patterns. Deposition yield is found to linearly scale with the flow rate, and duration of E-3D printing-DROPS. On the other hand, to prepare $2 \mathrm{H} \mathrm{MoS}_{2} / \mathrm{c}-\mathrm{rGO}$ $3 \mathrm{D} / 3 \mathrm{D}$ heterostructures HER electrodes, $1 \mathrm{~T} \mathrm{MoS}_{2} / \mathrm{c}-\mathrm{rGO} 3 \mathrm{D} / 3 \mathrm{D}$ heterostructures along with the supporting $\mathrm{CC}$ were annealed at $300^{\circ} \mathrm{C}$ for $2 \mathrm{~h}$ under ambient conditions. This post-annealing process ensures the phase transition from a distorted $1 \mathrm{~T}$ polymorph to an organized $2 \mathrm{H}$ phase. Note that no Nafion encapsulation coating or conductive paste was used to prepare the electrodes for HER. Further details of the methods are available in the Supplementary Information.

\section{Data availability}

The datasets generated during and/or analyzed during the current study are available from the corresponding author on reasonable request.

Received: 16 December 2019; Accepted: 16 June 2020; Published online: 15 July 2020

\section{References}

1. Frisenda, R. et al. Recent progress in the assembly of nanodevices and van der Waals heterostructures by deterministic placement of 2D materials. Chem. Soc. Rev. 47, 53-68 (2018)

2. Jariwala, D., Marks, T. J. \& Hersam, M. C. Mixed-dimensional van der Waals heterostructures. Nat. Mater. 16, 170 (2017).

3. Liu, Y., Huang, Y. \& Duan, X. Van der Waals integration before and beyond two-dimensional materials. Nature 567, 323 (2019).

4. Bae, S.-H. et al. Integration of bulk materials with two-dimensional materials for physical coupling and applications. Nat. Mater. 18, 550-560 (2019).

5. Li, S. et al. Vapour-liquid-solid growth of monolayer MoS2 nanoribbons. Nat. Mater. 17, 535 (2018).

6. Geim, A. K. \& Grigorieva, I. V. Van der Waals heterostructures. Nature 499 , 419-425 (2013).

7. Yang, Y., Li, G., Shrotriyal, V., Moriarty, T. \& Emery, K. High-efficiency solution processable self-organization of polymer blends. Nat. Mater. 4, 864-868 (2005).

8. Liu, Y. et al. Approaching the Schottky-Mott limit in van der Waals metalsemiconductor junctions. Nature 557, 697 (2018).

9. Kim, Y. et al. Remote epitaxy through graphene enables two-dimensional material-based layer transfer. Nature 544, 340 (2017).

10. Kong, W. et al. Polarity governs atomic interaction through two-dimensional materials. Nat. Mater. 17, 999 (2019).

11. Tung, V. C. et al. Surfactant-free water-processable photoconductive allcarbon composite. J. Am. Chem. Soc. 133, 4940 (2011).

12. Chen, Y.-C. et al. Structurally deformed MoS2 for electrochemically stable thermally resistant, and highly efficient hydrogen evolution reaction. Adv. Mater. 29, 1703863 (2017).

13. Qiu, L., Liu, J. Z., Chang, S. L. Y., Wu, Y. \& Li, D. Biomimetic superelastic graphene-based cellular monoliths. Nat. Commun. 3, 1241 (2012).

14. Israelachvili, J. N. Intermolecular and surface forces. (Elsevier, 2011).

15. Richard, D., Clanet, C. \& Quéré, D. Contact time of a bouncing drop. Nature 417, 811 (2002).

16. Qian, F. et al. Ultralight conductive silver nanowire aerogels. Nano Lett. 17, 7171-7176 (2017).

17. Estevez, L., Kelarakis, A., Gong, Q., Da’as, E. H. \& Giannelis, E. P. Multifunctional graphene/platinum/nafion hybrids via ice templating. J. Am. Chem. Soc 133, 6122-6125 (2011). 
18. Luo, J. et al. Compression and aggregation- resistant particles of crumpled soft. ACS Nano 5, 8943-8949 (2011).

19. Hattar, K., Bufford, D. C. \& Buller, D. L. Concurrent in situ ion irradiation transmission electron microscope. Nucl. Instrum Methods Phys. Res. Sect. B Beam Interact. with Mater. Atoms 338, 56-65 (2014).

20. Ii, R. C. W., Shi, T., Silva, E. C., Jovanovic, I. \& Robinson, J. A. Radiation effects on two-dimensional materials. Phys. Status Solidi a 213, 3065 (2016).

21. Atwater, H. A. et al. Materials challenges for the Starshot lightsail. Nat. Mater. 17, 861 (2018)

22. Yu, Y. et al. High phase-purity $1 \mathrm{~T}^{\prime}-\mathrm{MoS} 2-$ and $1 \mathrm{~T}^{\prime}-\mathrm{MoSe}$-layered crystals. Nat. Chem 10, 638-643 (2018).

23. Subbaraman, R. et al. Enhancing hydrogen evolution activity in water splitting by tailoring $\mathrm{Li}^{+}-\mathrm{Ni}(\mathrm{OH})_{2}-\mathrm{Pt}$ interfaces. Science 334, 1256-1260 (2011).

24. Subbaraman, R. et al. Trends in activity for the water electrolyser reactions on $3 \mathrm{~d} \mathrm{M}(\mathrm{Ni}, \mathrm{Co}, \mathrm{Fe}, \mathrm{Mn})$ hydr(oxy)oxide catalysts. Nat. Mater. 11, 550-557 (2012).

25. Davis, S. J. et al. Net-zero emissions energy systems. Science 360, 1419-1428 (2018).

26. Luo, Y. et al. Morphology and surface chemistry engineering toward $\mathrm{pH}-$ universal catalysts for hydrogen evolution at high current density. Nat. Commun. 10, 269 (2019).

27. $\mathrm{Hu}, \mathrm{J}$. et al. Nanohybridization of MoS 2 with layered double hydroxides efficiently synergizes the hydrogen evolution in alkaline media. Joule 1, 383 (2017).

28. Geng, X. et al. Three-dimensional structures of MoS2 nanosheets with ultrahigh hydrogen evolution reaction in water reduction. Adv. Funct. Mater. 24, 6123-6129 (2014).

29. $\mathrm{Pu}, \mathrm{Z}$. et al. 3D macroporous MoS2 thin film: In situ hydrothermal preparation and application as a highly active hydrogen evolution electrocatalyst at all pH values. Electrochim. Acta 168, 133 (2015).

30. Song, B. \& Jin, S. Two are better than one: heterostructures improve hydrogen evolution catalysis. Joule 1, 220 (2017).

31. Zhang, J. et al. Interface engineering of MoS2/Ni3S2heterostructures for highly enhanced electrochemical overall-water-splitting activity. Angew. Chemie-Int. Ed 55, 6702-6707 (2016).

32. Xiong, Q. et al. Cobalt covalent doping in MoS 2 to induce bifunctionality of overall water splitting. Adv. Mater. 30, 1801450 (2018).

33. Li, G. et al. Activating $\mathrm{MoS}_{2}$ for $\mathrm{pH}$-universal hydrogen evolution catalysis. J. Am. Chem. Soc. 139, 16194-16200 (2017).

34. Lukowski, M. A. et al. Enhanced hydrogen evolution catalysis from chemically exfoliated metallic MoS2 nanosheets. J. Am. Chem. Soc. 135, 10274-10277 (2013).

35. Yin, Y. et al. Synergistic phase and disorder engineering in $1 \mathrm{~T}-\mathrm{MoSe} 2$ nanosheets for enhanced hydrogen-evolution reaction. Adv. Mater. 29, 1-8 (2017).

36. Ding, Q., Song, B., Xu, P. \& Jin, S. Efficient electrocatalytic and photoelectrochemical hydrogen generation using MoS 2 and related compounds. Chem 1, 699-726 (2016).

37. Xie, J. et al. Controllable disorder engineering in oxygen-incorporated MoS 2 ultrathin nanosheets for efficient hydrogen evolution. J. Am. Chem. Soc. 135, 17881-17888 (2013).

38. Voiry, D. et al. Enhanced catalytic activity in strained chemically exfoliated WS2 nanosheets for hydrogen evolution. Nat. Mater. 12, 850-855 (2013).

39. Voiry, D. et al. Conducting $\mathrm{MoS}_{2}$ nanosheets as catalysts for hydrogen evolution reaction. Nano Lett. 13, 6222-6227 (2013).

40. Zang, Y. et al. Tuning orbital orientation endows molybdenum disulfide with exceptional alkaline hydrogen evolution capability. Nat. Commun. 10, 09210 (2019).

41. Huang, Y. et al. Atomically engineering activation sites onto metallic 1T-MoS 2 catalysts for enhanced electrochemical hydrogen evolution. Nat. Commun. 10, 08877 (2019).

42. Staszak-Jirkovský, J. et al. Design of active and stable Co-Mo-Sx chalcogels as $\mathrm{pH}$-universal catalysts for the hydrogen evolution reaction. Nat. Mater. 15, 197-203 (2015).

43. Duong, D. L., Yun, S. J. \& Lee, Y. H. van der Waals layered materials: opportunities and challenges. ACS Nano 11, 11803 (2017).

\section{Acknowledgements}

V.T., J.-H.F., and M.-H.C. are indebted to the support from the King Abdullah University of Science and Technology (KAUST) Office of Sponsored Research (OSR) under Award No: OSR-2018-CARF/CCF-3079. V.T. acknowledged the financial support from KAUST Catalysis Center (KCC). Characterization and fabrication of HER electrodes in this work were performed as User Proposals (\#5067 and \#5424) at the Molecular Foundry, Lawrence Berkeley National Lab, supported by the Office of Basic Energy Sciences, of the U.S. Department of Energy under Contract No. DE-AC02-05CH11231. Raman spectroscopy was performed at the Joint Center for Artificial Photosynthesis, a DOE Energy Innovation Hub, supported through the Office of Science of the U.S. Department of Energy under Award Number DE-SC0004993. A.Y.L. and J.K. also acknowledge the support from the KAUST under contract (OSR- 2015-CRG4-2634) and the support from U.S. Army Research Office through the MIT Institute for Soldier Nanotechnologies (Grant No. 023674). N.J.M and J.A.K acknowledge support from the U.S. Department of Energy, Office of Science under Award Number DE-SC0015894 and by a U.S. Department of Energy Office of Science Graduate Student Research (SCGSR) award. V.T. expresses his gratitude to Dr. Chang-Ming Jiang, Dr. Pin Lu, Wu Zhan, Vipawee Limsakoune, and Teresa L. Chen for the fruitful discussion in DROPS mechanisms and assistance in instrumentation. This work was performed, in part, at the Center for Integrated Nanotechnologies, an Office of Science User Facility operated for the U.S Department of Energy (DOE) Office of Science. Sandia National Laboratories is a multimission laboratory managed and operated by National Technology \& Engineering Solutions of Sandia, LLC, a wholly owned subsidiary of Honeywell International, Inc., for the U.S. DOE's National Nuclear Security Administration under contract DE-NA0003525. The views expressed in the article do not necessarily represent the views of the U.S. DOE or the United States Government.

\section{Author contributions}

V.T., L.J.L., and S.S.C. conceived and designed the experiments. J.H.F., C.C.W., Y.C.C., and A.Y.L. prepared the printed samples for SEM, XPS and Raman analyses. S.S.C. involved the EM imaging and characterization. N.J.M., J.A.K., and K.H. conducted the in-situ $\mathrm{I}^{3}$ TEM for ion irradiation. A.Y.L., M.H.C., L.J.L., and V.T. involved the analysis of HER data. V.T., S.S.C., L.J.L., J.K., J.A.K., and A.Y.L., were mainly responsible for preparing the paper with further inputs from other authors. All the authors discussed the results and commented on the paper.

\section{Competing interests}

The authors declare no competing interests.

\section{Additional information}

Supplementary information is available for this paper at https://doi.org/10.1038/s43246020-0041-2.

Correspondence and requests for materials should be addressed to V.T.

Reprints and permission information is available at http://www.nature.com/reprints

Publisher's note Springer Nature remains neutral with regard to jurisdictional claims in published maps and institutional affiliations.

Open Access This article is licensed under a Creative Commons Attribution 4.0 International License, which permits use, sharing, adaptation, distribution and reproduction in any medium or format, as long as you give appropriate credit to the original author(s) and the source, provide a link to the Creative Commons license, and indicate if changes were made. The images or other third party material in this article are included in the article's Creative Commons license, unless indicated otherwise in a credit line to the material. If material is not included in the article's Creative Commons license and your intended use is not permitted by statutory regulation or exceeds the permitted use, you will need to obtain permission directly from the copyright holder. To view a copy of this license, visit http://creativecommons.org/ licenses/by/4.0/

(c) The Author(s) 2020 